\title{
Combination Synchronization of Three Nonidentical Ecological Systems with Species Invasion
}

\author{
Xu Weigang $\mathbb{D}^{1,2}$ Yu Yilei $\mathbb{D}^{1,},^{1,2}$ and Wang Henian $\mathbb{D}^{1,2}$ \\ ${ }^{1}$ Institute of Wetland Research, Chinese Academy of Forestry, Beijing 100091, China \\ ${ }^{2}$ Beijing Key Laboratory of Wetland Services and Restoration, Beijing 100091, China \\ Correspondence should be addressed to Wang Henian; wanghn2006@hotmail.com
}

Received 22 December 2018; Revised 13 March 2019; Accepted 7 April 2019; Published 17 April 2019

Academic Editor: Ricardo Aguilar-Lopez

Copyright (c) $2019 \mathrm{Xu}$ Weigang et al. This is an open access article distributed under the Creative Commons Attribution License, which permits unrestricted use, distribution, and reproduction in any medium, provided the original work is properly cited.

\begin{abstract}
The research investigates synchronizing dynamics of three nonidentical ecological systems, one of which is invaded by an exterior top predator. For studying the effects of species invasion in the synchronizing dynamics, combination synchronization of one drive system and two response systems is designed: the ecological system with species invasion taken as drive system, and the other ecological systems as response systems. Using active backstepping design, the conditions for achievement of combination synchronization are derived. Under the conditions, numerical simulations are performed to demonstrate the synchronizing dynamics of ecological systems under species invasion. The results suggest that mechanism of synchronization plays an important role in determining chaos when ecological systems are interacting with each other.
\end{abstract}

\section{Introduction}

Chaos phenomena have been observed in many natural systems. As the research on chaos continues, great efforts were devoted to chaos synchronization of chaotic systems, a very important subfield in nonlinear science [1]. Since the pioneering work of Pecora and Carroll [2], chaos synchronization has been widely investigated in a variety of fields, such as ecological systems, chemical reactions, engineering science, and secure communication [3-8]. Many approaches have been proposed to achieve various types of chaos synchronization, for example, complete synchronization, phase synchronization, and generalized synchronization [1].

Most of the research works of chaos synchronization are limited to the synchronization of two chaotic systems, i.e., one drive system and one response system. Recently, Runzi et al. [9] developed a new type of synchronization, combination synchronization, which was applied to investigate the synchronization of three chaotic systems (two drive systems and one response system). From then, the synchronization of multiple chaotic systems was extensively studied. For example, Sun et al. [10] proposed a type of combinationcombination synchronization for four systems (two drive systems and two response systems).

The combination synchronization (or combinationcombination synchronization) can be possibly applied in the field of secure communication and information processing $[9,10]$. Sun et al. described that if synchronization can be controlled intelligently at will, then the combinationcombination synchronization may be possible to attain vastly better performance for secure communication and information processing. Ahmad et al. also exhibited an application of multi switching-combination synchronization control scheme in the case of secure communication [11]. Moreover, with the characteristics of synchronization of multiple systems, a possible application of combination synchronization can be realized in ecological field. For ecological systems are open systems, species interactions and species migration between ecological systems make the development of ecological systems often affected by other systems [12, 13]. After long-term evolution, the interactions between related ecological systems can bring about synchronization state of the systems [14]. 
In recent years, the complex dynamics of ecological systems, as an interesting and significant topic, is more and more concerned by the mathematicians and biologist. Via analyzing the nonlinear characteristics, many research works have played an important role in understanding the properties of natural ecosystems, such as basic structure, productivity, resource availability, interactions, stability, ecosystem size, and network connection. Systematical and detailed analysis on these characteristics can contribute to profound comprehension on population regulation, trophic relationships, energy transformation, and community behaviors in the ecological systems. Moreover, it can also promote the improvement as well as practical application of ecological theory. Emmerson and Dave provided a biological mechanism that explains the skewed distributions of interaction strengths in real communities, where emergent features of food webs reflect patterns of predator-prey body size $[15,16]$.

Since natural ecological systems are usually not isolated and closely connected with each other, the coupled networks of ecological systems can be more accurate to reflect the characteristics of nature and deserve further investigations. On the other hand, the coupled networks of ecological systems may exhibit more complex dynamic behaviors. Synchronization dynamics is one of the most interesting topics in the research where ecological systems are considered in connection. For example, Blasius and Stone studied the phase synchronization of two nonidentical diffusively predator-prey-resource systems and found that the synchronization patterns and travelling wave structures exhibited can correspond to those observed in natural systems [3]. Many research works show that the investigation on the synchronization of ecological chaotic systems is important for understanding the interactions between them and is valuable for ecological applications $[3,5,14,17]$.

Many evidences in field observation confirm the wide existence of synchronization between natural ecological systems. A classical example is the synchronization of Canada's hare-lynx cycle over millions of square kilometres [3]. This phenomenon has been documented for over 100 years. Also, northern vole populations in southeastern Norway exhibit large-scale, spatially synchronous population dynamics [18]. Motivated by the natural phenomena, a lot of theoretical works have been performed to investigate the synchronization in nature $[3,5,14,17]$. In the theoretical works, the synchronization of two ecological systems is often focused on. However, in many natural situations, the number of interacting systems may be three or more [19]. Therefore, it is necessary to make a development on the synchronization of three ecological systems, which is still not documented in existing literature.

Some researchers suggested that the achievement of ecological synchronization results from migration of species between ecological systems $[3,18]$. It is also described in literature that predatory mammals and birds can act as an agent of synchronization [5]. However, the possibility that species invasion, which widely exists in nature, can also drive the synchronization of ecological dynamics and is seldom considered. Since species invasion can pose great influences on the ecological systems and break the primary balance reached among local species, it is necessary to investigate the nonlinear dynamics determined by the synchronization of ecological systems, which is driven by the species invasion. This research focuses on the synchronization of three ecological systems when an exterior top predator invades into one of the three ecological systems via migration. The system with the invasive species is considered as drive system, and the other two systems are taken as response systems. The investigation on combination synchronization of the three systems can promote better comprehension on how the synchronization of ecological systems acts under species invasion.

In comparison with previous research works, the present study may exhibit three aspects of improvement in investigating the synchronization of chaotic ecological systems. Firstly, different from the former investigations which mainly focused on the complex dynamics and synchronization of two chaotic ecological systems, this research further tries to comprehend the synchronization among three chaotic ecological systems. Secondly, the three chaotic ecological systems considered are nonidentical and have different dimensions; therefore, the synchronization of the three systems is achieved through the interactions of complex dynamics induced by different ecological mechanisms. Thirdly, with the application of the method of combination synchronization, the case of exterior predator species simultaneously invading into two types of local environments is investigated. This may provide a new insight for studying the ecological dynamics of species invasion.

In this research, we apply active backstepping design to achieve the combination synchronization of the three ecological systems and numerical simulations of the combination synchronization are then performed, verifying the theoretical calculations. Following the former synchronization works of ecological systems, the combination synchronization in this research also demonstrates that one of the intrinsic mechanisms, predation (although the predation results from an exterior top predator), is a significant cause for population synchrony. Moreover, we find that, with the achievement of the combination synchronization, the invasion of predator species can force the extinction of local predator or dynamical transition between periodic and chaotic population oscillations. These nonlinear characteristics may help to guide the application in ecological engineering, such as harvesting or reduction of the invasive species.

\section{Systems Description}

Because of the importance of food chain in ecological systems, the food chain models are often employed in the approaches of synchronization of ecological systems $[3,5,14]$. Based on the literature, the food chain models are also used to study the combination synchronization of three ecological systems in this research.

Hastings and Powell [20] provided a classical dynamic model governed by a set of nonlinear ordinary differential equations to describe a tritrophic level food chain. The feeding relationships between the three species in the food 
chain are described in the following. Let $x$ denote the prey population at the lowest level of the food chain; population $x$ is predated by the individuals of population $y$; simultaneously, the population $y$ serves as a favorite food for individuals of population $z$, which is the top predator of the food chain. According to Hastings and Powell [20], the nondimensionalized form of the model system can be governed by

$$
\begin{aligned}
& \frac{d x}{d t}=x(1-x)-\frac{w_{1} x y}{1+w_{2} x} \\
& \frac{d y}{d t}=\frac{w_{1} x y}{1+w_{2} x}-\frac{w_{3} y z}{1+w_{4} y}-w_{5} y \\
& \frac{d z}{d t}=\frac{w_{3} y z}{1+w_{4} y}-w_{6} z
\end{aligned}
$$

in which $w_{1}, w_{2}, w_{3}, w_{4}, w_{5}$, and $w_{6}$ are parameters, and the interpretations of parameters' significance can be found in Hastings and Powell [20]. When biologically reasonable parameter values are chosen, system (1) can exhibit chaotic dynamics in long-term behavior.

In order to investigate synchronizing dynamics of ecological systems under the influences of species invasion, an exterior top predator $s$, which predates upon population $y$, is introduced into the food chain described above. Similarly to system (1), the new food chain model system with invasive top predator $s$ can be described as the following nonlinear differential equations:

$$
\begin{aligned}
& \frac{d x_{1}}{d t}=x_{1}\left(1-x_{1}\right)-\frac{w_{11} x_{1} y_{1}}{1+w_{12} x_{1}} \\
& \frac{d y_{1}}{d t}=\frac{w_{11} x_{1} y_{1}}{1+w_{12} x_{1}}-\frac{w_{13} y_{1} z_{1}}{1+w_{14} y_{1}}-\frac{w_{15} y_{1} s_{1}}{1+w_{16} y_{1}}-w_{17} y_{1} \\
& \frac{d z_{1}}{d t}=\frac{w_{13} y_{1} z_{1}}{1+w_{14} y_{1}}-w_{18} z_{1} \\
& \frac{d s_{1}}{d t}=\frac{w_{15} y_{1} s_{1}}{1+w_{16} y_{1}}-w_{19} s_{1}
\end{aligned}
$$

In system (2), the subscript " 1 " of $x, y, z$, and $s$ represents the first system of food chain considered for the combination synchronization, likewise in the below. The meaning of parameters $w_{11}, w_{12}, w_{13}, w_{14}, w_{17}$, and $w_{18}$ is the same as the corresponding parameters in system (1). Parameters $w_{15}$ and $w_{16}$ describe the predation relationship between population $s$ and population $y$; parameter $w_{19}$ represents the mortality rate of the population $s$.

Many researchers took into consideration the different functional responses for the tritrophic level food chain described by the Hastings-Powell model. In Naji and Balasim [21], they considered Beddington-DeAngelis functional response for the predation between population $x$ and population $y$, and between population $y$ and population $z$ in the three-species food chain. The nondimensionalized form of this model system can be described by

$$
\begin{aligned}
& \frac{d x_{2}}{d t}=x_{2}\left(1-x_{2}\right)-\frac{x_{2} y_{2}}{w_{21} y_{2}+x_{2}+w_{22}} \\
& \frac{d y_{2}}{d t}=\frac{w_{23} x_{2} y_{2}}{w_{21} y_{2}+x_{2}+w_{22}}-\frac{y_{2} z_{2}}{w_{24} z_{2}+y_{2}+w_{25}}-w_{26} y_{2} \\
& \frac{d z_{2}}{d t}=\frac{w_{27} y_{2} z_{2}}{w_{24} z_{2}+y_{2}+w_{25}}-w_{28} z_{2}
\end{aligned}
$$

The significance of parameters $w_{21}, w_{22}, w_{23}, w_{24}, w_{25}, w_{26}$, $w_{27}$, and $w_{28}$ of the system (3) was described in Naji and Balasim [21].

For the tritrophic level food chain which has the prey-intermediate predator-top predator structure, Upadhyay et al. [22] proposed a food chain model which consists of a hybrid type of Holling type-II with Beddington-DeAngelis type of functional responses. Population $y$ feeds on population $x$ according to Holling type-II functional response, whereas population $z$ predates upon population $y$ according to Beddington-DeAngelis type functional response. Moreover, the dynamics of the top predator $z$ is described by the dynamic equation developed by Upadhyay and Rai [23]. This food chain model can be governed by the following nondimensionalized system of nonlinear differential equations.

$$
\begin{aligned}
& \frac{d x_{3}}{d t}=x_{3}\left(1-x_{3}\right)-\frac{x_{3} y_{3}}{x_{3}+w_{31}} \\
& \frac{d y_{3}}{d t}=\frac{w_{32} x_{3} y_{3}}{x_{3}+w_{33}}-\frac{y_{3} z_{3}}{w_{34} z_{3}+y_{3}+w_{35}}-w_{36} y_{3} \\
& \frac{d z_{3}}{d t}=w_{37} z_{3}^{2}-\frac{w_{38} z_{3}^{2}}{y_{3}+w_{39}}
\end{aligned}
$$

The interpretation of the parameters of the system (4), $w_{31}$, $w_{32}, w_{33}, w_{34}, w_{35}, w_{36}, w_{37}, w_{38}$, and $w_{39}$, was explained in Upadhyay et al. [22].

The combination synchronization of the systems (2), (3), and (4) is investigated in this research. In order to achieve the combination synchronization of the three systems, the following synchronization strategy is designed.

\section{Combination Synchronization of Systems (2), (3), and (4)}

According to the description of ecological systems in the above section, the invasive species $s$ can directly change the dynamics of $x, y$, and $z$ in system (2) and indirectly influence the dynamics of systems (3) and (4). In order to investigate the combination synchronization driven by invasive species $s$, the ecological system which is directly involved with the invasive species, i.e., system (2), is considered as the drive system, and systems (3) and (4) are considered as the response systems. And then the combination synchronization between the drive system (2) and the two response systems (3) and (4) is investigated. Based on previous research works, the definition 
of such combination synchronization can be described as the following.

Definition $1([6,7])$. Combination synchronization of the drive system (2) and the response systems (3) and (4) is said to be achieved, if there exist three nonzero matrices $A \in \mathrm{R}^{3} \times \mathrm{R}^{4}$, $B \in \mathrm{R}^{3} \times \mathrm{R}^{3}$, and $C \in \mathrm{R}^{3} \times \mathrm{R}^{3}$ such that

$$
\lim _{t \rightarrow+\infty}\left\|B\left(\begin{array}{l}
x_{2} \\
y_{2} \\
z_{2}
\end{array}\right)+C\left(\begin{array}{l}
x_{3} \\
y_{3} \\
z_{3}
\end{array}\right)-A\left(\begin{array}{l}
x_{1} \\
y_{1} \\
z_{1} \\
s_{1}
\end{array}\right)\right\|=0
$$

where $\|\cdot\|$ denotes Euclidean norm. The constant matrices $A$, $B$, and $C$ are called the scaling matrices.

Let the response systems be controlled, and the controlled systems (3) and (4) can be described by

$$
\begin{aligned}
\frac{d x_{2}}{d t}= & x_{2}\left(1-x_{2}\right)-\frac{x_{2} y_{2}}{w_{21} y_{2}+x_{2}+w_{22}}+u_{21} \\
\frac{d y_{2}}{d t}= & \frac{w_{23} x_{2} y_{2}}{w_{21} y_{2}+x_{2}+w_{22}}-\frac{y_{2} z_{2}}{w_{24} z_{2}+y_{2}+w_{25}}-w_{26} y_{2} \\
& +u_{22} \\
\frac{d z_{2}}{d t}= & \frac{w_{27} y_{2} z_{2}}{w_{24} z_{2}+y_{2}+w_{25}}-w_{28} z_{2}+u_{23}
\end{aligned}
$$

and

$$
\begin{aligned}
& \frac{d x_{3}}{d t}=x_{3}\left(1-x_{3}\right)-\frac{x_{3} y_{3}}{x_{3}+w_{31}}+u_{31} \\
& \frac{d y_{3}}{d t}=\frac{w_{32} x_{3} y_{3}}{x_{3}+w_{33}}-\frac{y_{3} z_{3}}{w_{34} z_{3}+y_{3}+w_{35}}-w_{36} y_{3}+u_{32} \\
& \frac{d z_{3}}{d t}=w_{37} z_{3}^{2}-\frac{w_{38} z_{3}^{2}}{y_{3}+w_{39}}+u_{33}
\end{aligned}
$$

in which $u_{21}, u_{22}, u_{23}, u_{31}, u_{32}$, and $u_{33}$ are the controllers to be designed.

According to Runzi et al. [9], the achievement of combination synchronization of the three systems is determined by stability of the origin point of an error system. Therefore firstly, the error system for the drive system (2) and response systems (5) and (6) need to be generated. Let

$$
\left(\begin{array}{l}
e_{1} \\
e_{2} \\
e_{3}
\end{array}\right)=\left(\begin{array}{cccc}
a_{1} & 0 & 0 & 0 \\
0 & a_{2} & 0 & 0 \\
0 & 0 & a_{3} & a_{4}
\end{array}\right)\left(\begin{array}{l}
x_{1} \\
y_{1} \\
z_{1} \\
s_{1}
\end{array}\right)
$$

$$
\begin{array}{r}
-\left(\begin{array}{lll}
b_{1} & 0 & 0 \\
0 & b_{2} & 0 \\
0 & 0 & b_{3}
\end{array}\right)\left(\begin{array}{l}
x_{2} \\
y_{2} \\
z_{2}
\end{array}\right) \\
-\left(\begin{array}{lll}
c_{1} & 0 & 0 \\
0 & c_{2} & 0 \\
0 & 0 & c_{3}
\end{array}\right)\left(\begin{array}{l}
x_{3} \\
y_{3} \\
z_{3}
\end{array}\right)
\end{array}
$$

in which $a_{i}, b_{i}$, and $c_{i}$ are parameters of combination synchronization. Calculating the derivatives for $e_{1}, e_{2}$, and $e_{3}$, we have

$$
\begin{aligned}
& \frac{d e_{1}}{d t}=\left(1-\frac{1}{w_{21}}\right) e_{1}-\frac{a_{1} w_{11}}{a_{2} w_{12}} e_{2}+\frac{1}{w_{21}}\left(a_{1} x_{1}-c_{1} x_{3}\right) \\
& -\frac{a_{1} w_{11}}{a_{2} w_{12}}\left(b_{2} y_{2}+c_{2} y_{3}\right)-a_{1} x_{1}^{2}+b_{1} x_{2}^{2}+c_{1} x_{3}^{2} \\
& +\frac{a_{1} w_{11}}{w_{12}} \frac{y_{1}}{1+w_{12} x_{1}}-\frac{b_{1}}{w_{21}} \frac{x_{2}\left(x_{2}+w_{22}\right)}{w_{21} y_{2}+x_{2}+w_{22}} \\
& +c_{1} \frac{x_{3} y_{3}}{x_{3}+w_{31}}-b_{1} u_{21}-c_{1} u_{31} \\
& \frac{d e_{2}}{d t}=\frac{b_{2} w_{23}}{b_{1} w_{21}} e_{1}+\left(\frac{w_{11}}{w_{12}}-\frac{1}{w_{24}}-w_{17}\right) e_{2} \\
& -\left(\frac{a_{2} w_{13}}{a_{3} w_{14}}+\frac{a_{2} w_{15}}{a_{4} w_{16}}\right) e_{3} \\
& -\frac{b_{2} w_{23}}{b_{1} w_{21}}\left(a_{1} x_{1}-c_{1} x_{3}\right)+\frac{w_{11}}{w_{12}}\left(b_{2} y_{2}+c_{2} y_{3}\right) \\
& +\frac{1}{w_{24}}\left(a_{2} y_{1}-c_{2} y_{3}\right)+b_{2}\left(w_{26}-w_{17}\right) y_{2} \\
& +c_{2}\left(w_{36}-w_{17}\right) y_{3}+\frac{a_{2} a_{3} w_{15}}{a_{4} w_{16}} z_{1} \\
& -\left(\frac{a_{2} w_{13}}{a_{3} w_{14}}+\frac{a_{2} w_{15}}{a_{4} w_{16}}\right)\left(b_{3} z_{2}+c_{3} z_{3}\right) \\
& +\frac{a_{2} a_{4} w_{13}}{a_{3} w_{14}} s_{1}-\frac{a_{2} w_{11}}{w_{12}} \frac{y_{1}}{1+w_{12} x_{1}} \\
& +\frac{a_{2} w_{13}}{w_{14}} \frac{z_{1}}{1+w_{14} y_{1}} \\
& +\frac{b_{2} w_{23}}{w_{21}} \frac{x_{2}\left(x_{2}+w_{22}\right)}{w_{21} y_{2}+x_{2}+w_{22}} \\
& -\frac{b_{2}}{w_{24}} \frac{y_{2}\left(y_{2}+w_{25}\right)}{w_{24} z_{2}+y_{2}+w_{25}}+\frac{a_{2} w_{15}}{w_{16}} \frac{s_{1}}{1+w_{16} y_{1}} \\
& -c_{2} w_{32} \frac{x_{3} y_{3}}{x_{3}+w_{33}}+c_{2} \frac{y_{3} z_{3}}{w_{34} z_{3}+y_{3}+w_{35}} \\
& -b_{2} u_{22}-c_{2} u_{32} \\
& \frac{d e_{3}}{d t}=\frac{b_{3} w_{27}}{b_{2} w_{24}} e_{2}+\left(\frac{w_{13}}{w_{14}}+\frac{w_{15}}{w_{16}}-w_{19}\right) e_{3}
\end{aligned}
$$




$$
\begin{aligned}
& +\frac{2 w_{37}}{c_{3}}\left(a_{3} z_{1}+a_{4} s_{1}-b_{3} z_{2}\right) e_{3}-\frac{w_{37}}{c_{3}} e_{3}^{2} \\
& -\frac{b_{3} w_{27}}{b_{2} w_{24}}\left(a_{2} y_{1}-c_{2} y_{3}\right) \\
& +a_{3}\left(w_{19}-w_{18}-\frac{w_{15}}{w_{16}}\right) z_{1} \\
& +b_{3}\left(w_{28}-w_{19}\right) z_{2}-c_{3} w_{19} z_{3} \\
& +\left(\frac{w_{13}}{w_{14}}+\frac{w_{15}}{w_{16}}\right)\left(b_{3} z_{2}+c_{3} z_{3}\right)-\frac{a_{4} w_{13}}{w_{14}} s_{1} \\
& -\frac{w_{37}}{c_{3}}\left(a_{3} z_{1}+a_{4} s_{1}-b_{3} z_{2}\right)^{2} \\
& -\frac{a_{3} w_{13}}{w_{14}} \frac{z_{1}}{1+w_{14} y_{1}} \\
& +\frac{b_{3} w_{27}}{w_{24}} \frac{y_{2}\left(y_{2}+w_{25}\right)}{w_{24} z_{2}+y_{2}+w_{25}} \\
& -\frac{a_{4} w_{15}}{w_{16}} \frac{s_{1}}{1+w_{16} y_{1}}+c_{3} w_{38} \frac{z_{3}^{2}}{y_{3}+w_{39}}-b_{3} u_{23} \\
& -c_{3} u_{33}
\end{aligned}
$$

For convenience, we define the combination controllers as follows:

$$
\begin{aligned}
& U_{1}=b_{1} u_{21}+c_{1} u_{31} \\
& U_{2}=b_{2} u_{22}+c_{2} u_{32} \\
& U_{3}=b_{3} u_{23}+c_{3} u_{33}
\end{aligned}
$$

Then the error system can be further described as

$$
\begin{aligned}
\frac{d e_{1}}{d t}= & \left(1-\frac{1}{w_{21}}\right) e_{1}-\frac{a_{1} w_{11}}{a_{2} w_{12}} e_{2}+f-U_{1} \\
\frac{d e_{2}}{d t}= & \frac{b_{2} w_{23}}{b_{1} w_{21}} e_{1}+\left(\frac{w_{11}}{w_{12}}-\frac{1}{w_{24}}-w_{17}\right) e_{2} \\
& -\left(\frac{a_{2} w_{13}}{a_{3} w_{14}}+\frac{a_{2} w_{15}}{a_{4} w_{16}}\right) e_{3}+g-U_{2} \\
\frac{d e_{3}}{d t}= & \frac{b_{3} w_{27}}{b_{2} w_{24}} e_{2}+\left(\frac{w_{13}}{w_{14}}+\frac{w_{15}}{w_{16}}-w_{19}\right) e_{3} \\
& +\frac{2 w_{37}}{c_{3}}\left(a_{3} z_{1}+a_{4} s_{1}-b_{3} z_{2}\right) e_{3}-\frac{w_{37}}{c_{3}} e_{3}^{2}+h \\
& -U_{3}
\end{aligned}
$$

in which

$$
\begin{aligned}
f= & \frac{1}{w_{21}}\left(a_{1} x_{1}-c_{1} x_{3}\right)-\frac{a_{1} w_{11}}{a_{2} w_{12}}\left(b_{2} y_{2}+c_{2} y_{3}\right)-a_{1} x_{1}^{2} \\
& +b_{1} x_{2}^{2}+c_{1} x_{3}^{2}+\frac{a_{1} w_{11}}{w_{12}} \frac{y_{1}}{1+w_{12} x_{1}}
\end{aligned}
$$

$$
\begin{aligned}
& -\frac{b_{1}}{w_{21}} \frac{x_{2}\left(x_{2}+w_{22}\right)}{w_{21} y_{2}+x_{2}+w_{22}}+c_{1} \frac{x_{3} y_{3}}{x_{3}+w_{31}} \\
& g=-\frac{b_{2} w_{23}}{b_{1} w_{21}}\left(a_{1} x_{1}-c_{1} x_{3}\right)+\frac{w_{11}}{w_{12}}\left(b_{2} y_{2}+c_{2} y_{3}\right) \\
& +\frac{1}{w_{24}}\left(a_{2} y_{1}-c_{2} y_{3}\right)+b_{2}\left(w_{26}-w_{17}\right) y_{2} \\
& +c_{2}\left(w_{36}-w_{17}\right) y_{3}+\frac{a_{2} a_{3} w_{15}}{a_{4} w_{16}} z_{1} \\
& -\left(\frac{a_{2} w_{13}}{a_{3} w_{14}}+\frac{a_{2} w_{15}}{a_{4} w_{16}}\right)\left(b_{3} z_{2}+c_{3} z_{3}\right) \\
& +\frac{a_{2} a_{4} w_{13}}{a_{3} w_{14}} s_{1}-\frac{a_{2} w_{11}}{w_{12}} \frac{y_{1}}{1+w_{12} x_{1}} \\
& +\frac{a_{2} w_{13}}{w_{14}} \frac{z_{1}}{1+w_{14} y_{1}}+\frac{b_{2} w_{23}}{w_{21}} \frac{x_{2}\left(x_{2}+w_{22}\right)}{w_{21} y_{2}+x_{2}+w_{22}} \\
& -\frac{b_{2}}{w_{24}} \frac{y_{2}\left(y_{2}+w_{25}\right)}{w_{24} z_{2}+y_{2}+w_{25}}+\frac{a_{2} w_{15}}{w_{16}} \frac{s_{1}}{1+w_{16} y_{1}} \\
& -c_{2} w_{32} \frac{x_{3} y_{3}}{x_{3}+w_{33}}+c_{2} \frac{y_{3} z_{3}}{w_{34} z_{3}+y_{3}+w_{35}} \\
& h=-\frac{b_{3} w_{27}}{b_{2} w_{24}}\left(a_{2} y_{1}-c_{2} y_{3}\right)+a_{3}\left(w_{19}-w_{18}-\frac{w_{15}}{w_{16}}\right) z_{1} \\
& +b_{3}\left(w_{28}-w_{19}\right) z_{2}-c_{3} w_{19} z_{3} \\
& +\left(\frac{w_{13}}{w_{14}}+\frac{w_{15}}{w_{16}}\right)\left(b_{3} z_{2}+c_{3} z_{3}\right)-\frac{a_{4} w_{13}}{w_{14}} s_{1} \\
& -\frac{w_{37}}{c_{3}}\left(a_{3} z_{1}+a_{4} s_{1}-b_{3} z_{2}\right)^{2} \\
& -\frac{a_{3} w_{13}}{w_{14}} \frac{z_{1}}{1+w_{14} y_{1}}+\frac{b_{3} w_{27}}{w_{24}} \frac{y_{2}\left(y_{2}+w_{25}\right)}{w_{24} z_{2}+y_{2}+w_{25}} \\
& -\frac{a_{4} w_{15}}{w_{16}} \frac{s_{1}}{1+w_{16} y_{1}}+c_{3} w_{38} \frac{z_{3}^{2}}{y_{3}+w_{39}}
\end{aligned}
$$

We need to find the combination controllers $U_{1}, U_{2}, U_{3}$ to make the systems (2), (3), and (4) achieve combination synchronization. The combination synchronization of the three systems demands that the error system has a global asymptotically stable equilibrium $(0,0,0)^{\mathrm{T}}$. In order to obtain such controllers, the following steps are taken.

(1) First let

$$
v_{1}=e_{1}
$$

and

$$
U_{1}=f
$$

Then we have

$$
\frac{d v_{1}}{d t}=\left(1-\frac{1}{w_{21}}\right) v_{1}-\frac{a_{1} w_{11}}{a_{2} w_{12}} e_{2}
$$


Consider the $\left\{v_{1}\right\}$-subsystem, and choose the following Lyapunov function for this subsystem:

$$
V_{1}=\frac{1}{2} v_{1}^{2}
$$

The derivative of the Lyapunov function (16) is

$$
\frac{d V_{1}}{d t}=v_{1}\left(\left(1-\frac{1}{w_{21}}\right) v_{1}-\frac{a_{1} w_{11}}{a_{2} w_{12}} e_{2}\right)
$$

In order to stabilize the $\left\{v_{1}\right\}$-subsystem, let $e_{2}$ be a virtual controller

$$
e_{2}=p\left(v_{1}\right)=\frac{a_{2} w_{12}}{a_{1} w_{11}}\left(2-\frac{1}{w_{21}}\right) v_{1}
$$

Then we have

$$
\frac{d V_{1}}{d t}=-v_{1}^{2} \leq 0
$$

(2) Let

$$
v_{2}=e_{2}-p\left(v_{1}\right)
$$

Combining (20) with (11), (13), and (15), we have the following $\left\{v_{1}, v_{2}\right\}$-subsystem:

$$
\begin{aligned}
\frac{d v_{1}}{d t} & =-v_{1}-\frac{a_{1} w_{11}}{a_{2} w_{12}} v_{2} \\
\frac{d v_{2}}{d t} & =\left(\frac{b_{2} w_{23}}{b_{1} w_{21}}\right. \\
& \left.+\frac{a_{2} w_{12}}{a_{1} w_{11}}\left(2-\frac{1}{w_{21}}\right)\left(\frac{w_{11}}{w_{12}}-\frac{1}{w_{24}}-w_{17}+1\right)\right) v_{1} \\
& +\left(\frac{w_{11}}{w_{12}}-\frac{1}{w_{21}}-\frac{1}{w_{24}}-w_{17}+2\right) v_{2}-\left(\frac{a_{2} w_{13}}{a_{3} w_{14}}\right. \\
& \left.+\frac{a_{2} w_{15}}{a_{4} w_{16}}\right) e_{3}+g-U_{2}
\end{aligned}
$$

Consider the following Lyapunov function for the $\left\{v_{1}, v_{2}\right\}$ subsystem:

$$
V_{2}=\frac{1}{2} v_{1}^{2}+\frac{1}{2} v_{2}^{2}
$$

And the derivative of (22) is

$$
\begin{aligned}
& \frac{d V_{2}}{d t}=-v_{1}^{2}+\left(\frac{b_{2} w_{23}}{b_{1} w_{21}}-\frac{a_{1} w_{11}}{a_{2} w_{12}}\right. \\
& \left.+\frac{a_{2} w_{12}}{a_{1} w_{11}}\left(2-\frac{1}{w_{21}}\right)\left(\frac{w_{11}}{w_{12}}-\frac{1}{w_{24}}-w_{17}+1\right)\right) \\
& \cdot v_{1} v_{2}+\left(\frac{w_{11}}{w_{12}}-\frac{1}{w_{21}}-\frac{1}{w_{24}}-w_{17}+2\right) v_{2}^{2} \\
& \quad-\left(\frac{a_{2} w_{13}}{a_{3} w_{14}}+\frac{a_{2} w_{15}}{a_{4} w_{16}}\right) v_{2} e_{3}+v_{2}\left(g-U_{2}\right)
\end{aligned}
$$

In order to make the $\left\{v_{1}, v_{2}\right\}$-subsystem asymptotically approach $(0,0)$, we define

$$
e_{3}=q\left(v_{1}, v_{2}\right)=0
$$

and

$$
\begin{aligned}
U_{2} & =\left(\frac{b_{2} w_{23}}{b_{1} w_{21}}-\frac{a_{1} w_{11}}{a_{2} w_{12}}\right. \\
& \left.+\frac{a_{2} w_{12}}{a_{1} w_{11}}\left(2-\frac{1}{w_{21}}\right)\left(\frac{w_{11}}{w_{12}}-\frac{1}{w_{24}}-w_{17}+1\right)\right) v_{1} \\
& +\left(w_{32}-w_{37}-\frac{1}{w_{24}}-\frac{1}{w_{21}}+3\right) v_{2}+g
\end{aligned}
$$

Therefore,

$$
\frac{d V_{2}}{d t}=-v_{1}^{2}-v_{2}^{2} \leq 0
$$

which suggests the $\left\{v_{1}, v_{2}\right\}$-subsystem is asymptotical stable.

(3) Let

$$
v_{3}=e_{3}-q\left(v_{1}, v_{2}\right)
$$

Combining (23) with (11), (13), (20), and (21), we can have $\left\{v_{1}, v_{2}, v_{3}\right\}$-subsystem as follows:

$$
\begin{aligned}
\frac{d v_{1}}{d t}= & -v_{1}-\frac{a_{1} w_{11}}{a_{2} w_{12}} v_{2} \\
\frac{d v_{2}}{d t}= & \frac{a_{1} w_{11}}{a_{2} w_{12}} v_{1}-v_{2}-\left(\frac{a_{2} w_{13}}{a_{3} w_{14}}+\frac{a_{2} w_{15}}{a_{4} w_{16}}\right) v_{3} \\
\frac{d v_{3}}{d t}= & \frac{a_{2} b_{3} w_{12} w_{27}}{a_{1} b_{2} w_{11} w_{24}}\left(2-\frac{1}{w_{21}}\right) v_{1}+\frac{b_{3} w_{27}}{b_{2} w_{24}} v_{2} \\
& +\left(\frac{w_{13}}{w_{14}}+\frac{w_{15}}{w_{16}}-w_{19}\right) v_{3} \\
& +\frac{2 w_{37}}{c_{3}}\left(a_{3} z_{1}+a_{4} s_{1}-b_{3} z_{2}\right) v_{3}-\frac{w_{37}}{c_{3}} v_{3}^{2} \\
& +h-U_{3}
\end{aligned}
$$

Consider the following Lyapunov function for the $\left\{v_{1}, v_{2}, v_{3}\right\}$ subsystem:

$$
V_{3}=\frac{1}{2} v_{1}^{2}+\frac{1}{2} v_{2}^{2}+\frac{1}{2} v_{3}^{2}
$$

Thus

$$
\begin{aligned}
& \frac{d V_{3}}{d t}=-v_{1}^{2}-v_{2}^{2}+\frac{a_{2} b_{3} w_{12} w_{27}}{a_{1} b_{2} w_{11} w_{24}}\left(2-\frac{1}{w_{21}}\right) v_{1} v_{3} \\
& +\left(\frac{b_{3} w_{27}}{b_{2} w_{24}}-\frac{a_{2} w_{13}}{a_{3} w_{14}}-\frac{a_{2} w_{15}}{a_{4} w_{16}}\right) v_{2} v_{3}+\left(\frac{w_{13}}{w_{14}}+\frac{w_{15}}{w_{16}}\right. \\
& \left.\quad-w_{19}+\frac{2 w_{37}}{c_{3}}\left(a_{3} z_{1}+a_{4} s_{1}-b_{3} z_{2}\right)\right) v_{3}^{3}-\frac{w_{37}}{c_{3}} v_{3}^{3} \\
& +v_{3}\left(h-U_{3}\right)
\end{aligned}
$$


Let

$$
\begin{aligned}
U_{3} & =\frac{a_{2} b_{3} w_{12} w_{27}}{a_{1} b_{2} w_{11} w_{24}}\left(2-\frac{1}{w_{21}}\right) v_{1}+\left(\frac{b_{3} w_{27}}{b_{2} w_{24}}-\frac{a_{2} w_{13}}{a_{3} w_{14}}\right. \\
& \left.-\frac{a_{2} w_{15}}{a_{4} w_{16}}\right) v_{2}-\frac{w_{37}}{c_{3}} v_{3}^{3}+\left(\frac{w_{13}}{w_{14}}+\frac{w_{15}}{w_{16}}-w_{19}+1\right. \\
& \left.+\frac{2 w_{37}}{c_{3}}\left(a_{3} z_{1}+a_{4} s_{1}-b_{3} z_{2}\right)\right) v_{3}+h
\end{aligned}
$$

Then the derivative of the Lyapunov function of the $\left\{v_{1}, v_{2}, v_{3}\right\}$-subsystem can always satisfy

$$
\frac{d V_{3}}{d t}=-v_{1}^{2}-v_{2}^{2}-v_{3}^{3} \leq 0
$$

According to Lyapunov stability theorem, the equilibrium $(0,0,0)$ is global asymptotically stable for the $\left\{v_{1}, v_{2}, v_{3}\right\}$ subsystem. Moreover, since

$$
\left(\begin{array}{l}
e_{1} \\
e_{2} \\
e_{3}
\end{array}\right)=\left(\begin{array}{ccc}
1 & 0 & 0 \\
\frac{a_{2} w_{12}}{a_{1} w_{11}}\left(2-\frac{1}{w_{21}}\right) & 1 & 0 \\
0 & 0 & 1
\end{array}\right)\left(\begin{array}{l}
v_{1} \\
v_{2} \\
v_{3}
\end{array}\right)
$$

it can be concluded for the error system (11) that

$$
\left(\begin{array}{l}
e_{1} \\
e_{2} \\
e_{3}
\end{array}\right) \longrightarrow 0, \text { as } t \longrightarrow+\infty
$$

under the combination controllers described in (14), (25), and (31). It means that the systems (2), (3), and (4) achieve synchronization combination. Summarizing the above calculations, we have the following theorem.

Theorem 2. If the combination control laws are chosen as follows:

$$
\begin{aligned}
U_{1} & =f \\
U_{2} & =\left(\frac{b_{2} w_{23}}{b_{1} w_{21}}-\frac{a_{1} w_{11}}{a_{2} w_{12}}\right. \\
& \left.+\frac{a_{2} w_{12}}{a_{1} w_{11}}\left(2-\frac{1}{w_{21}}\right)\left(\frac{w_{11}}{w_{12}}-\frac{1}{w_{24}}-w_{17}+1\right)\right) \\
& \cdot\left(a_{1} x_{1}-b_{1} x_{2}-c_{1} x_{3}\right)+g+\left(w_{32}-w_{37}-\frac{1}{w_{24}}\right. \\
& \left.-\frac{1}{w_{21}}+3\right)\left(a_{2} y_{1}-b_{2} y_{2}-c_{2} y_{3}\right. \\
& \left.-\frac{a_{2} w_{12}}{a_{1} w_{11}}\left(2-\frac{1}{w_{21}}\right)\left(a_{1} x_{1}-b_{1} x_{2}-c_{1} x_{3}\right)\right) \\
U_{3} & =\frac{a_{2} b_{3} w_{12} w_{27}}{a_{1} b_{2} w_{11} w_{24}}\left(2-\frac{1}{w_{21}}\right)\left(a_{1} x_{1}-b_{1} x_{2}-c_{1} x_{3}\right) \\
& -\frac{w_{37}}{c_{3}}\left(a_{3} z_{1}+a_{4} s_{1}-b_{3} z_{2}-c_{3} z_{3}\right)^{3}+\left(\frac{b_{3} w_{27}}{b_{2} w_{24}}\right.
\end{aligned}
$$

$$
\begin{aligned}
& \left.-\frac{a_{2} w_{13}}{a_{3} w_{14}}-\frac{a_{2} w_{15}}{a_{4} w_{16}}\right)\left(a_{2} y_{1}-b_{2} y_{2}-c_{2} y_{3}\right. \\
& \left.-\frac{a_{2} w_{12}}{a_{1} w_{11}}\left(2-\frac{1}{w_{21}}\right)\left(a_{1} x_{1}-b_{1} x_{2}-c_{1} x_{3}\right)\right) \\
& +\left(\frac{w_{13}}{w_{14}}+\frac{w_{15}}{w_{16}}-w_{19}+1\right. \\
& \left.+\frac{2 w_{37}}{c_{3}}\left(a_{3} z_{1}+a_{4} s_{1}-b_{3} z_{2}\right)\right)\left(a_{3} z_{1}+a_{4} s_{1}-b_{3} z_{2}\right. \\
& \left.-c_{3} z_{3}\right)+h
\end{aligned}
$$

then the drive system (2) will achieve combination synchronization with the response systems (3) and (4).

\section{Numerical Results}

Numerical simulations are performed to demonstrate the combination synchronization of the systems (2), (3), and (4). Fourth-order Runge-Kutta method is applied with time step equal to 0.001 . For the parameters of combination synchronization described in (7), we assume that $a_{i}=b_{j}=c_{j}=1 \quad(i=1,2$, $3,4$ and $j=1,2,3)$.

It is necessary to describe the dynamic behaviors with given feasible parameter values for the three systems involved in the combination synchronization. The dynamics of the three systems has been studied a lot in literature. According to Naji and Balasim [21], when

$$
\begin{aligned}
& w_{21}=0.3, \\
& w_{22}=0.2, \\
& w_{23}=0.8, \\
& w_{24}=0.2, \\
& w_{25}=0.2, \\
& w_{26}=0.2, \\
& w_{27}=0.2, \\
& w_{28}=0.01
\end{aligned}
$$

the system (3) follows chaotic behavior. While $0.82<w_{23}<$ 0.86 with other parameter values fixed, system (3) exhibits periodic behavior.

The system (4) also shows chaotic behavior when the parameter values satisfy the following:

$$
\begin{aligned}
& w_{31}=0.48, \\
& w_{32}=2.93, \\
& w_{33}=0.54, \\
& 0.01<w_{34}<0.29, \\
& w_{35}=0.1, \\
& w_{36}=1.15, \\
& w_{37}=0.35,
\end{aligned}
$$



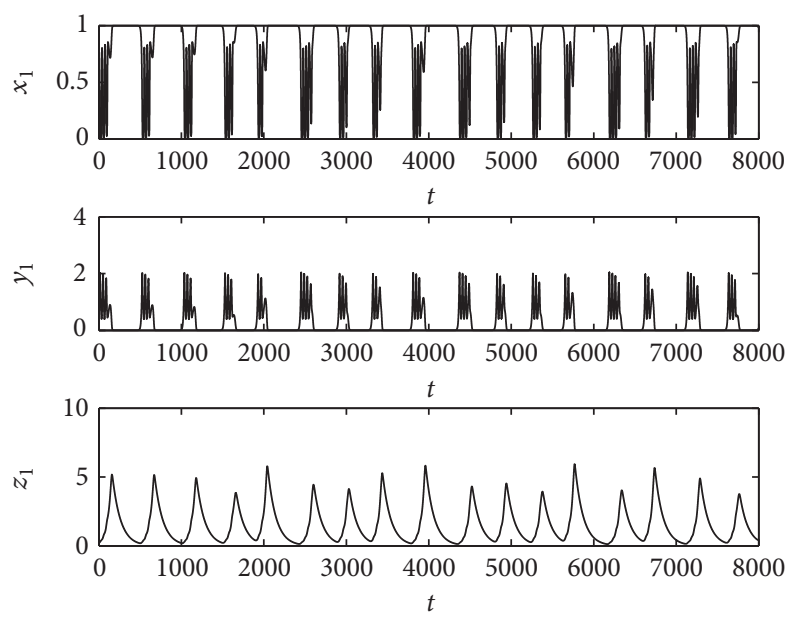

(a)
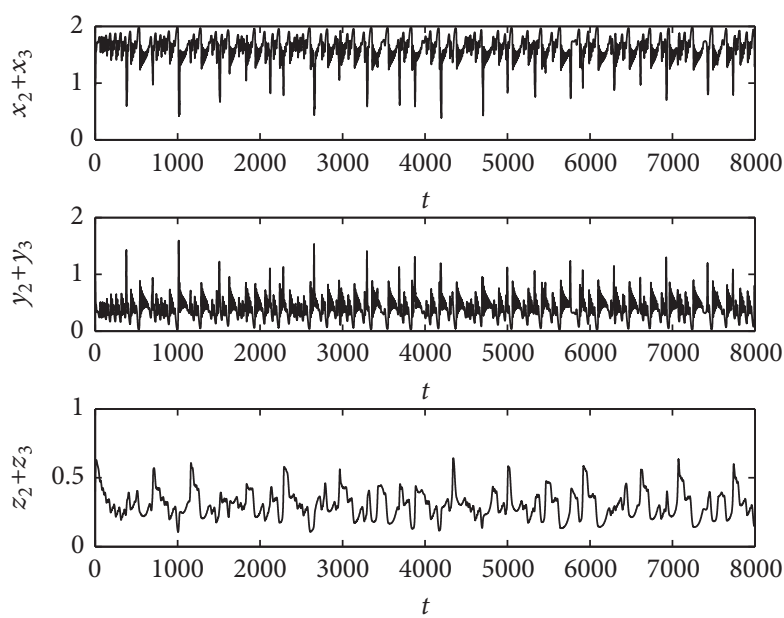

(c)

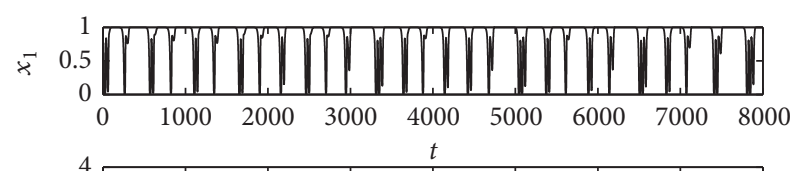

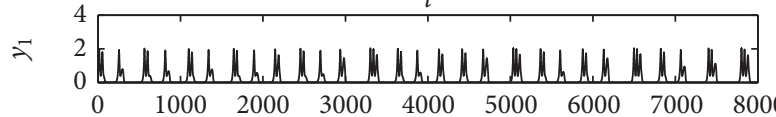

$\overrightarrow{0}$

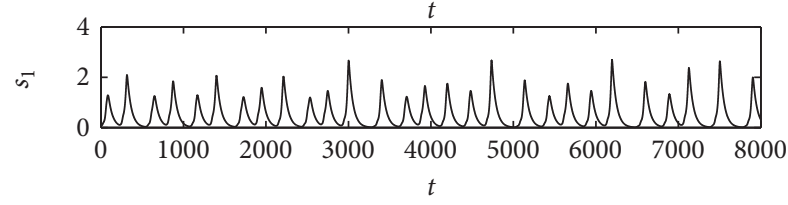

(b)
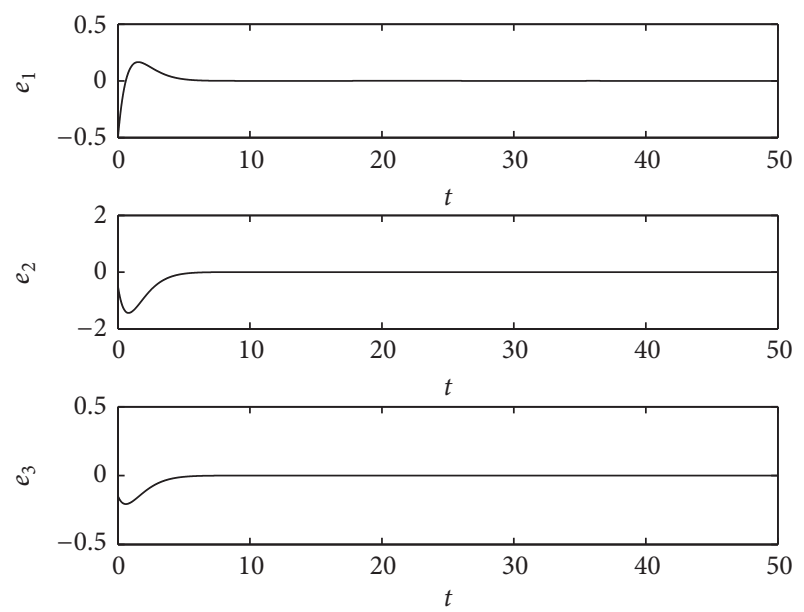

(d)

Figure 1: Combination synchronization of systems (2), (3), and (4). The four graphs describe the time involution of state vectors of system (2) (without and with invasive species), (a) and (b); state vectors of combined systems of (3) and (4), $x_{2}+x_{3}, y_{2}+y_{3}, z_{2}+z_{3}$, (c); and state vectors of error system, $e_{1}, e_{2}, e_{3},(\mathrm{~d}) . w_{15}=0.2, w_{16}=2, w_{19}=0.02, w_{34}=0.2$, and the other parameters are given in $(36) \sim(38)$.

$$
\begin{aligned}
& w_{38}=0.2, \\
& w_{39}=0.25
\end{aligned}
$$

according to the research work of Upadhyay et al. [22]. When $0.29<w_{34}<0.56$ as well as other parameter values fixed, periodic behavior takes place.

As for the system (2), when there is no invasive species, the feasible value for showing chaotic behavior can be given as

$$
\begin{aligned}
& w_{11}=1, \\
& w_{12}=3, \\
& w_{13}=0.1, \\
& w_{14}=2, \\
& w_{17}=0.1, \\
& w_{18}=0.01
\end{aligned}
$$

System (2) shows periodic behavior when the parameter value of $w_{13}$ changes to be 0.2 . The three parameters related to the invasive species, $w_{15}, w_{16}$, and $w_{19}$, are kept as varying parameters to study the combination synchronization of systems (2), (3), and (4) in different cases.

The initial states of populations $x, y, z$ for the drive system and the responses systems are given the same as $x(0)=0.5$, $y(0)=0.5, z(0)=0.2$. As for the population $s$, two cases of initial states, $s(0)=0$ and $s(0)=0.05$, are selected, so that the dynamics of the three ecological systems before and after species invasion can be compared. Moreover, given the above initial states, the initial states for the error system can be obtained, $e_{1}(0)=-0.5, e_{2}(0)=-0.5$, and $e_{3}(0)=-0.15$.

The numerical results of combination synchronization are depicted in Figures 1 and 2. Figure 1 shows the case where system (2) (without invasive species), system (3), and system (4) all exhibit chaotic oscillation. As shown in Figure 1(b), the invasion of species $s$ results in the increase of frequencies of the chaotic oscillations of the other populations in system 

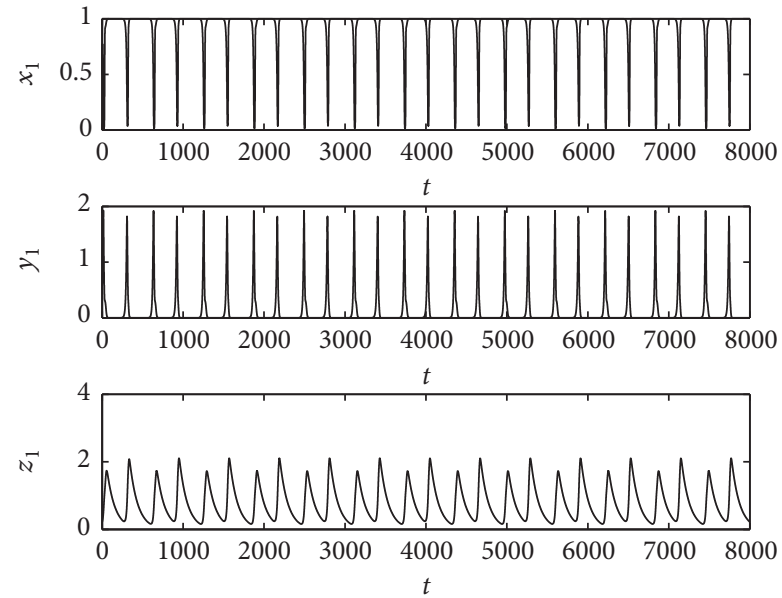

(a)

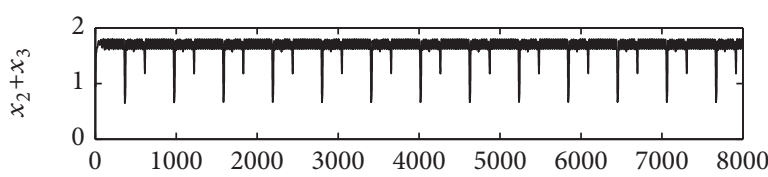

$t$
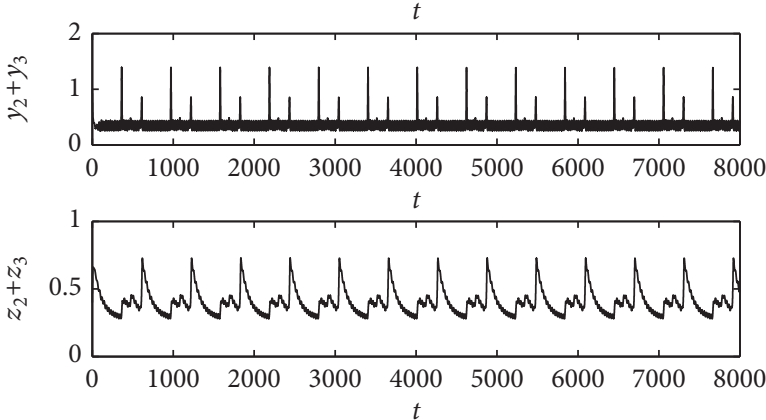

(c)
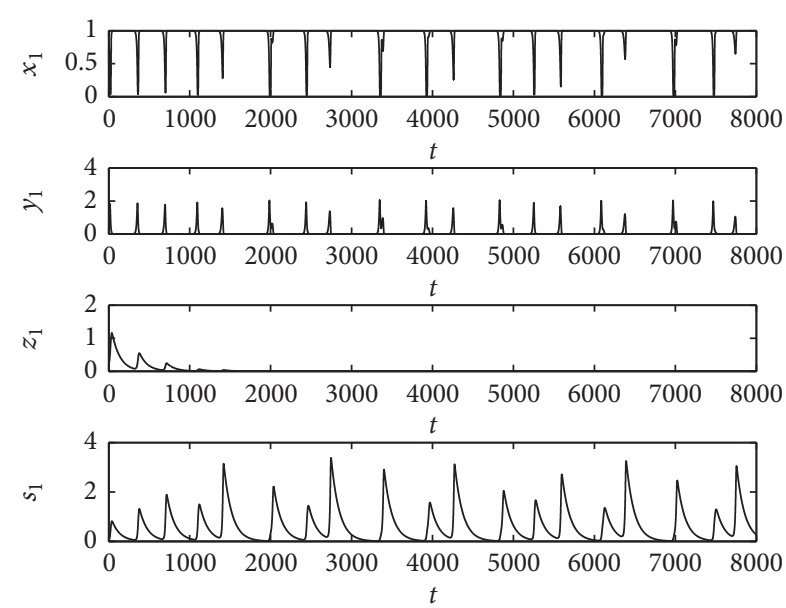

(b)
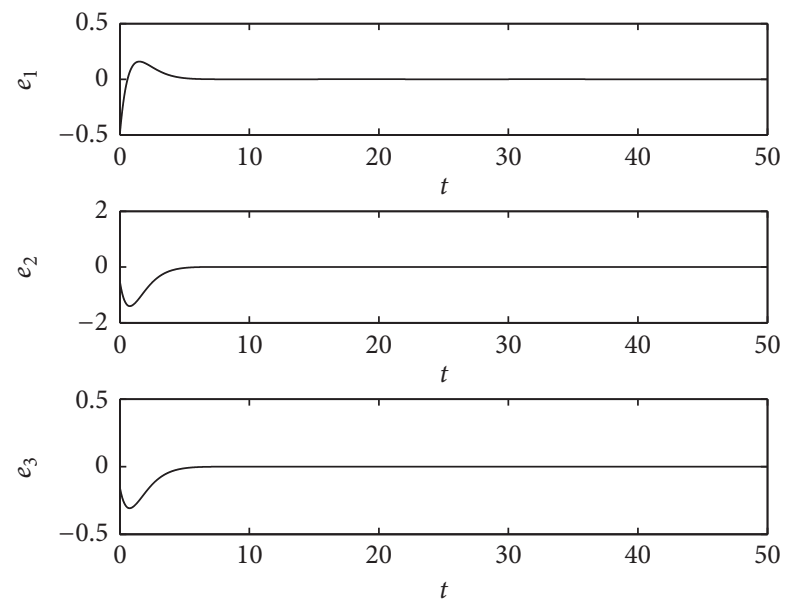

(d)

Figure 2: Combination synchronization of systems (2), (3), and (4) for the case in which systems (2) (without invasive species), (3), and (4) exhibit periodic oscillations. Likewise in Figure 1, similar four graphs for various state vectors are plotted. $w_{13}=0.2, w_{15}=0.25, w_{16}=1.5$, $w_{19}=0.01, w_{23}=0.85, w_{34}=0.4$, and the other parameters are given in $(36) \sim(38)$.

(2). Figure 1(d) shows the convergence of error system to the equilibrium $(0,0,0)^{\mathrm{T}}$ as time progresses. That means the three systems achieve combination synchronization after the species invasion. Moreover, via the combination synchronization, the invasive species can even force the dynamics of the two response systems to change into new chaotic oscillations shown in the drive system.

Figure 2 shows another case of combination synchronization where systems (2) (without invasive species), (3), and (4) show periodic oscillations (Figures 2(a) and 2(c)). As shown in Figure 2(b), the invasive species $s$ not only eliminates the primary top predator $z$, but also forces the system (2) to evolve into chaotic oscillation. The asymptotical approaching to $(0,0,0)^{\mathrm{T}}$ shown in Figure 2(d) suggests the achievement of combination synchronization, via which the extinction of population $z$ and the chaotic oscillation can also be extended to systems (3) and (4).

Figure 3 demonstrates that the combination synchronization results in a dynamical transition from periodic to chaotic behaviors. As shown in Figures 3(a) and 3(b), the limit cycles in the phase portraits suggest that systems (3) and (4) exhibit periodic oscillations of the populations, which are also verified by the sensitivity analysis in Figures 3(d) and $3(\mathrm{e})$ showing periodic waves with tiny amplitudes. The amplitudes of such periodic waves change proportionally to the difference between the two initial conditions applied in sensitivity analysis. However, after combination synchronization among the systems (2), (3), and (4), the invasive species forces the two response systems to turn into chaotic behavior, as demonstrated by the strange attractor in Figure 3(c) and verified by the sensitivity analysis in Figure 3(e) showing aperiodic and irregular waves with amplitude much larger than the difference of initial conditions. Actually, the amplitude of such waves can also reach this level in Figure 3(e) even if the two initial conditions for sensitivity analysis are set to be closer, such as $10^{-8}$ or smaller. Therefore, it suggests the dynamical behavior in Figure 3(c) is sensitive to initial conditions, an important property of chaos. 


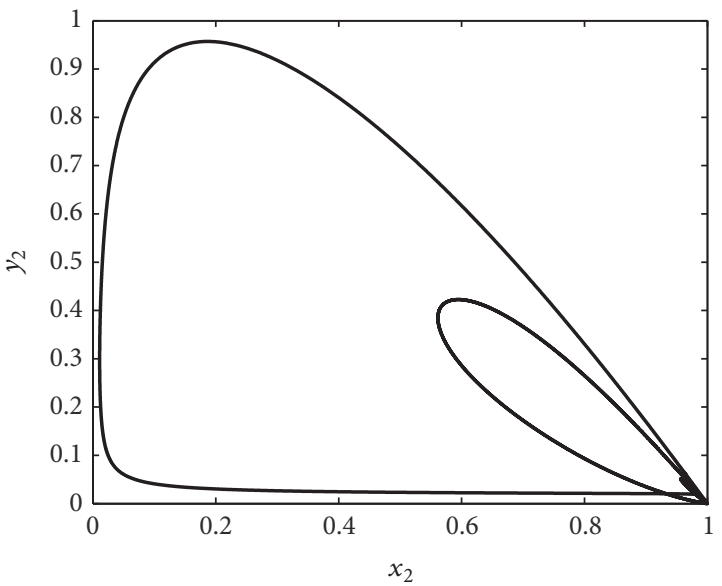

(a)

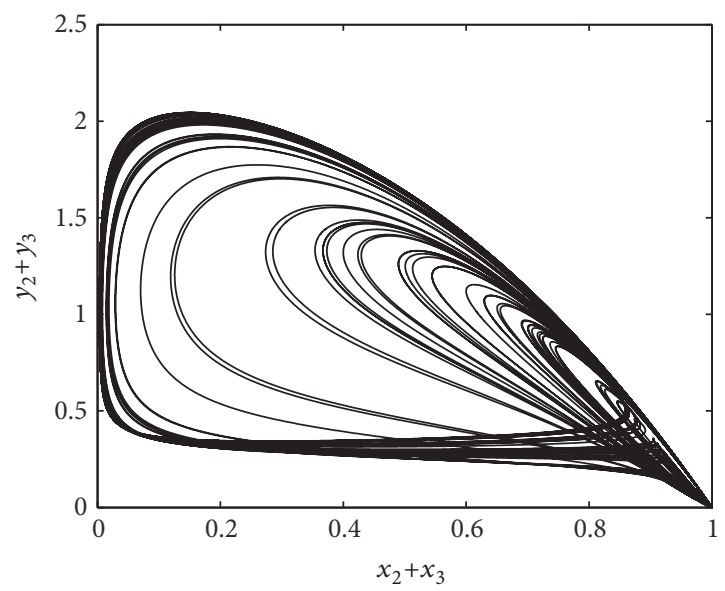

(c)
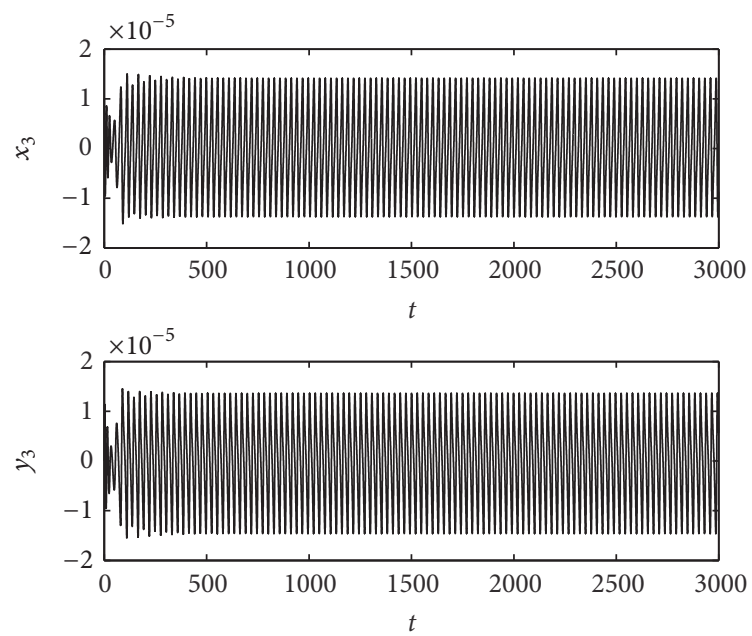

(e)

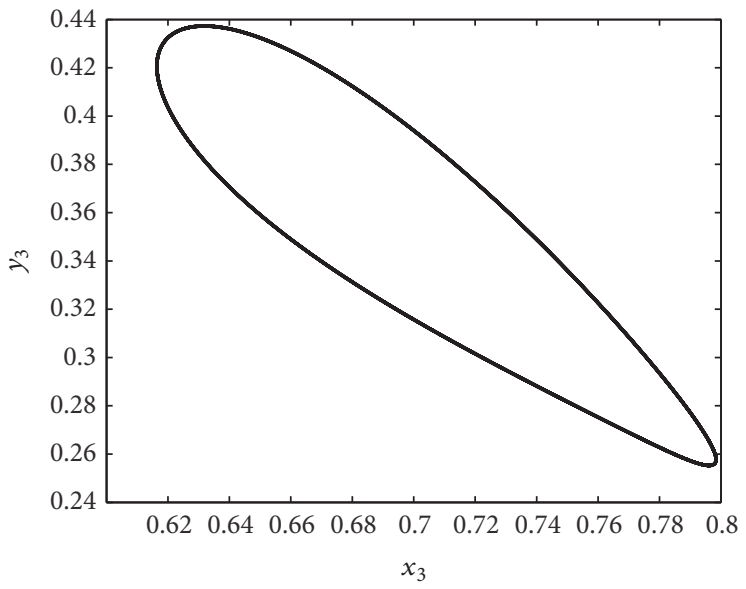

(b)
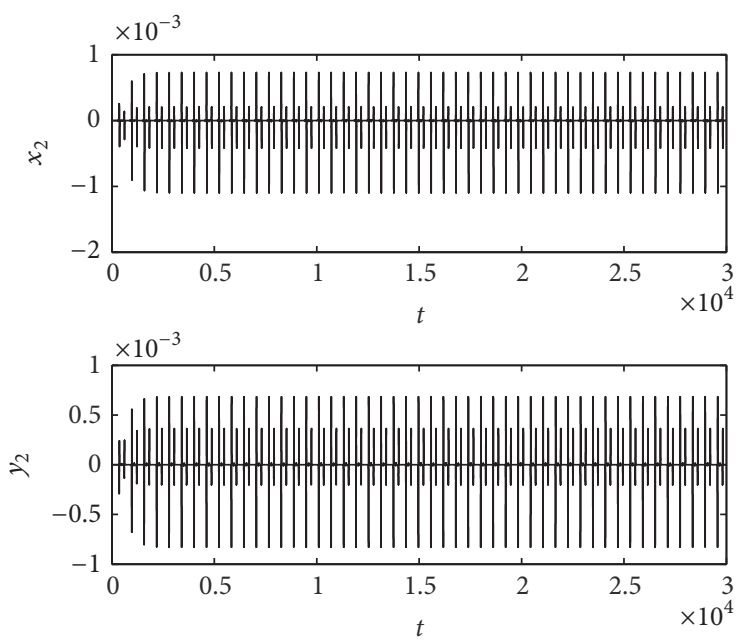

(d)
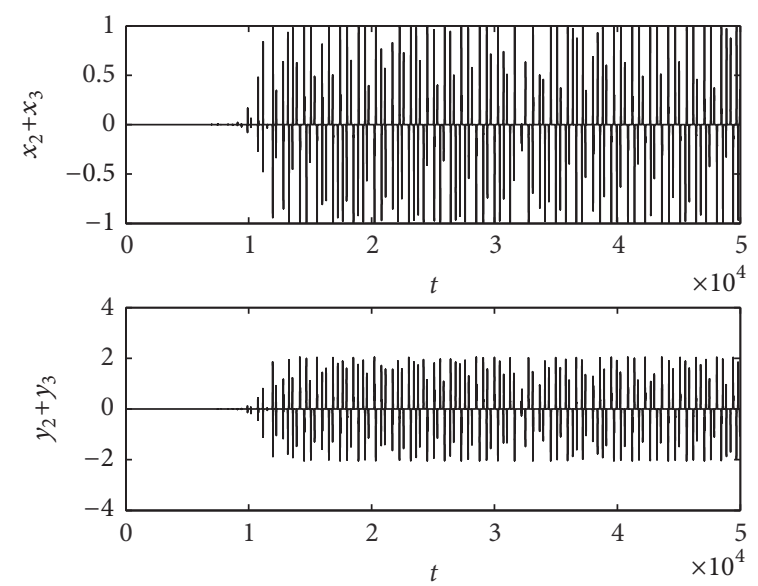

(f)

Figure 3: Dynamics of systems (3) and (4) before and after combination synchronization. The graphs are $x_{2}-y_{2}$ and $x_{3}-y_{3}$ phase portraits before the combination synchronization, (a) and (b); phase portrait after the combination synchronization, (c); sensitivity analysis on the dynamics corresponding to upper row, (d), (e), and (f). The sensitivity analysis is performed by comparing the difference of dynamics when two close initial conditions, which show difference of $10^{-5}$, are applied. The parametric conditions are the same as those in Figure 2. 


\section{Discussion and Conclusions}

Nowadays, many ecological systems suffer from the problem of species invasion [24-26]. Species invasion can greatly alter the population dynamics of the ecological system invaded. Moreover, via the interactions between the ecological systems, the dynamics of the related ecological systems can also be forced to change. As time progresses, the primary balance among ecological systems will be broken and new synchronization state may be achieved.

To study ecological mechanisms for such phenomenon, the combination synchronization of three ecological systems is investigated in this research. Each ecological system is described by a food chain and one of the three systems is considered to suffer from species invasion of an exterior top predator. Taking ecological system with species invasion as drive system, and the other two systems as response systems, the conditions for combination synchronization of the three systems are derived using active backstepping design. Under the conditions, numerical simulations of combination synchronization are performed. With the results obtained from numerical simulations, the following should be addressed:

(1) The achievement of combination synchronization makes the variation of population dynamics, which results from species invasion, expand from drive system to response systems.

(2) Under species invasion and achievement of combination synchronization, the frequencies of population oscillations of the three ecological systems can be greatly changed.

(3) For the dynamics of response systems, the achievement of combination synchronization can lead to the transition from periodic behaviors to chaotic behaviors. For the case where the two response systems exhibit periodic oscillation, the achievement of combination synchronization can force the chaotic dynamics of the drive system extending to the response systems (see Figure 2). Such dynamical transition is also revealed by many previous research works [27-29]. It interprets the chaos induced by synchronization of ecological systems.

(4) The invasion of exterior top predator and achievement of combination synchronization can bring about the extinction of local predator in all three ecological systems.

The combination synchronization research of three ecological systems provides an insight into the complex dynamics due to interactions of ecological systems. Moreover, the conclusions described above may be able to extend to the synchronizing dynamics of more than three ecological systems, in which one is drive system and three or more are response systems. For the case where the numbers of drive system and response system are both larger than two, combination-combination synchronization can be further considered to study the complex dynamics based on the results obtained here.

\section{Data Availability}

The data of numerical results are generated during the study.

\section{Conflicts of Interest}

The authors declare no conflicts of interest.

\section{Acknowledgments}

This research was financed by the Fundamental Research Funds for the Central Non-profit Research Institution of CAF (No. CAFINT2014C15).

\section{References}

[1] S. Boccaletti, J. Kurths, G. Osipov, D. L. Valladares, and C. S. Zhou, "The synchronization of chaotic systems," Physics Reports, vol. 366, no. 1-2, pp. 1-101, 2002.

[2] L. M. Pecora and T. L. Carroll, "Synchronization in chaotic systems," Physical Review Letters, vol. 64, no. 8, pp. 821-824, 1990.

[3] B. Blasius and L. Stone, "Chaos and phase synchronization in ecological systems," International Journal of Bifurcation and Chaos, vol. 10, no. 10, pp. 2361-2380, 2000.

[4] H.-K. Chen, "Global chaos synchronization of new chaotic systems via nonlinear control," Chaos, Solitons and Fractals, vol. 23, no. 4, pp. 1245-1251, 2005.

[5] R. K. Upadhyay and V. Rai, "Complex dynamics and synchronization in two non-identical chaotic ecological systems," Chaos, Solitons and Fractals, vol. 40, no. 5, pp. 2233-2241, 2009.

[6] L. Gámez-Guzmán, C. Cruz-Hernández, R. M. LópezGutiérrez, and E. E. García-Guerrero, "Synchronization of Chua's circuits with multi-scroll attractors: application to communication," Communications in Nonlinear Science and Numerical Simulation, vol. 14, no. 6, pp. 2765-2775, 2009.

[7] K. M. Cuomo and A. V. Oppenheim, "Paper 11 - circuit implementation of synchronized chaos with applications to communications," Controlling Chaos, vol. 71, no. 1, pp. 153-156, 1996.

[8] Z.-P. Jiang, "A note on chaotic secure communication systems," IEEE Transactions on Circuits and Systems I: Fundamental Theory and Applications, vol. 49, no. 1, pp. 92-96, 2002.

[9] L. Runzi, W. Yinglan, and D. Shucheng, "Combination synchronization of three classic chaotic systems using active backstepping design," Chaos: An Interdisciplinary Journal of Nonlinear Science, vol. 21, no. 4, article no. 043114, 2011.

[10] J. Sun, Y. Shen, G. Zhang, C. Xu, and G. Cui, "Combinationcombination synchronization among four identical or different chaotic systems," Nonlinear Dynamics, vol. 73, no. 3, pp. 12111222, 2013.

[11] I. Ahmad, M. Shafiq, and M. M. Al-Sawalha, "Globally exponential multi switching-combination synchronization control of chaotic systems for secure communications," Chinese Journal of Physics, vol. 56, no. 3, pp. 974-987, 2018.

[12] M. Loreau and N. Mouquet, "Immigration and the maintenance of local species diversity," The American Naturalist, vol. 154, no. 4, pp. 427-440, 1999.

[13] J. H. Brown, T. G. Whitham, S. K. Morgan Ernest, and C. A. Gehring, "Complex species interactions and the dynamics of 
ecological systems: long-term experiments," Science, vol. 293, no. 5530, pp. 643-650, 2001.

[14] B. Blasius, A. Huppert, and L. Stone, "Complex dynamics and phase synchronization in spatially extended ecological systems," Nature, vol. 399, no. 6734, pp. 354-359, 1999.

[15] M. C. Emmerson and D. Raffaelli, "Predator-prey body size, interaction strength and the stability of a real food web," Journal of Animal Ecology, vol. 73, no. 3, pp. 399-409, 2004.

[16] M. P. David and T. Gaku, "Proximate mechanisms for variation in food-chain length," Oikos, vol. 116, no. 5, pp. 775-782, 2007.

[17] X.-J. Wu, J. Li, and R. K. Upadhyay, "Chaos control and synchronization of a three-species food chain model via Holling functional response," International Journal of Computer Mathematics, vol. 87, no. 1-3, pp. 199-214, 2010.

[18] R. A. Ims and H. P. Andreassen, "Spatial synchronization of vole population dynamics by predatory birds," Nature, vol. 408, no. 6809, pp. 194-196, 2000.

[19] M. D. Holland and A. Hastings, "Strong effect of dispersal network structure on ecological dynamics," Nature, vol. 456, no. 7223, pp. 792-795, 2008.

[20] A. Hastings and T. Powell, "Chaos in a three-species food chain," Ecology, vol. 72, no. 3, pp. 896-903, 1991.

[21] R. K. Naji and A. T. Balasim, "Dynamical behavior of a three species food chain model with Beddington-DeAngelis functional response," Chaos, Solitons \& Fractals, vol. 32, no. 5, pp. 1853-1866, 2007.

[22] R. K. Upadhyay, R. K. Naji, S. N. Raw, and B. Dubey, “The role of top predator interference on the dynamics of a food chain model," Communications in Nonlinear Science and Numerical Simulation, vol. 18, no. 3, pp. 757-768, 2013.

[23] R. K. Upadhyay and V. Rai, "Why chaos is rarely observed in natural populations," Chaos, Solitons \& Fractals, vol. 8, no. 12, pp. 1933-1939, 1997.

[24] R. K. Didham, J. M. Tylianakis, N. J. Gemmell, T. A. Rand, and R. M. Ewers, "Interactive effects of habitat modification and species invasion on native species decline," Trends in Ecology \& Evolution, vol. 22, no. 9, pp. 489-496, 2007.

[25] O. Honnay, K. Verheyen, and M. Hermy, "Permeability of ancient forest edges for weedy plant species invasion," Forest Ecology and Management, vol. 161, no. 1-3, pp. 109-122, 2002.

[26] J. T. Lehman and C. E. Cáceres, "Food-web responses to species invasion by a predatory invertebrate: bythotrephes in lake michigan," Limnology and Oceanography, vol. 38, no. 4, pp. 879891, 1993.

[27] W. Gilpin and M. W. Feldman, "A phase transition induces chaos in a predator-prey ecosystem with a dynamic fitness landscape," PLoS Computational Biology, vol. 13, no. 7, article no. e1005644, 2017.

[28] A. Arellano-Delgado, R. M. López-Gutiérrez, M. A. MurilloEscobar, L. Cardoza-Avendaño, and C. Cruz-Hernández, "The emergence of hyperchaos and synchronization in networks with discrete periodic oscillators," Entropy, vol. 19, no. 8, aricle no. 413, 2017.

[29] C.-N. Wang, J. Ma, Y. Liu, and L. Huang, "Chaos control, spiral wave formation, and the emergence of spatiotemporal chaos in networked Chua circuits," Nonlinear Dynamics, vol. 67, no. 1, pp. 139-146, 2012. 


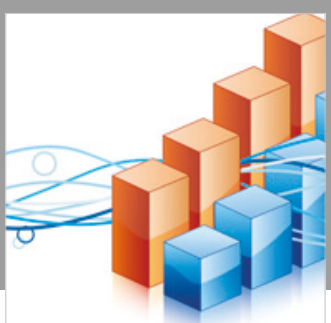

Advances in

Operations Research

\section{-n-m}
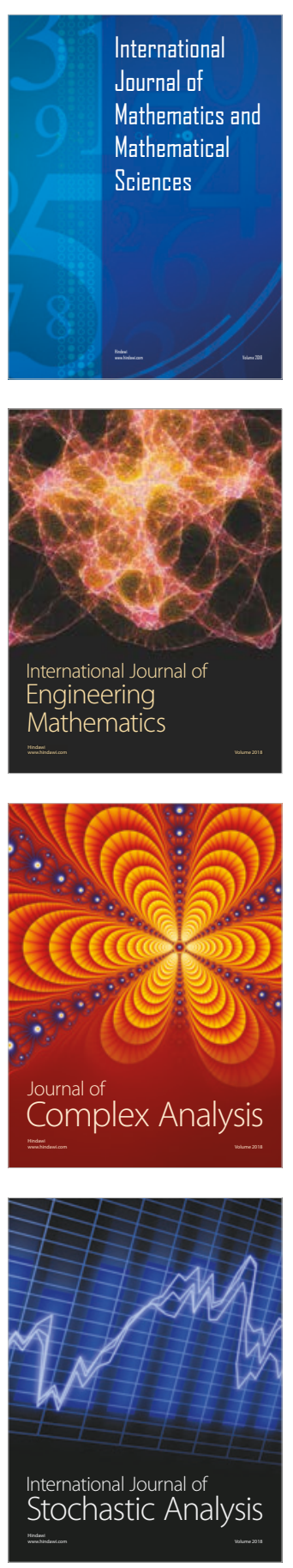
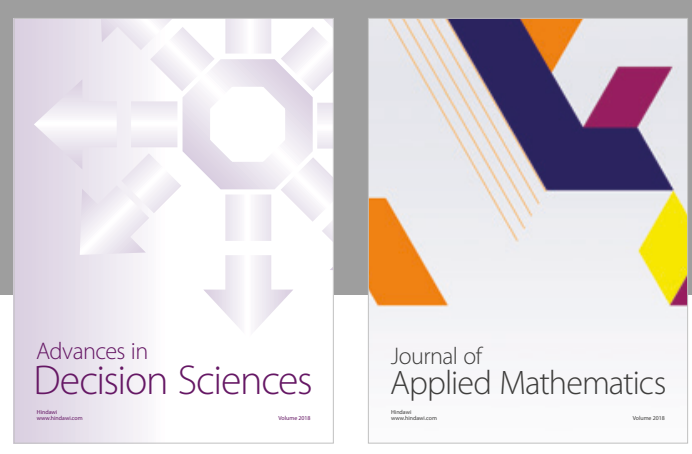

Journal of

Applied Mathematics
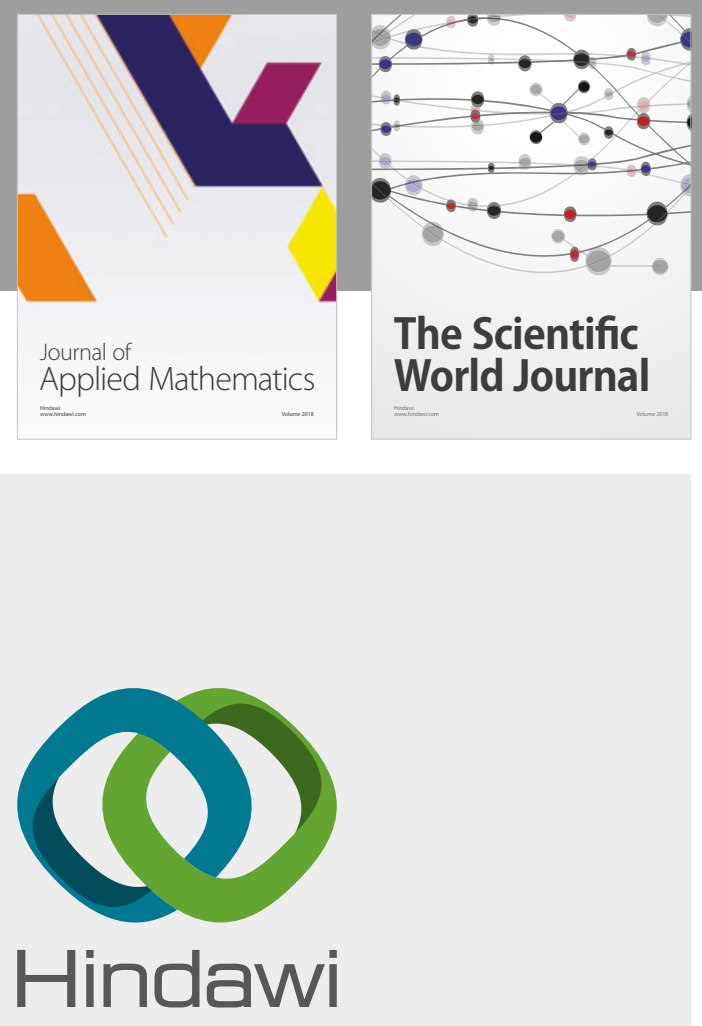

Submit your manuscripts at

www.hindawi.com

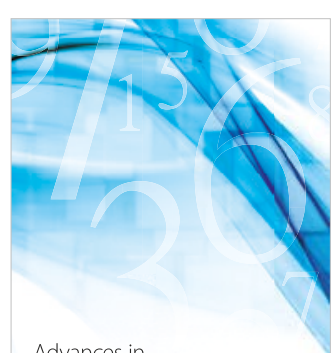

Advances in
Numerical Analysis
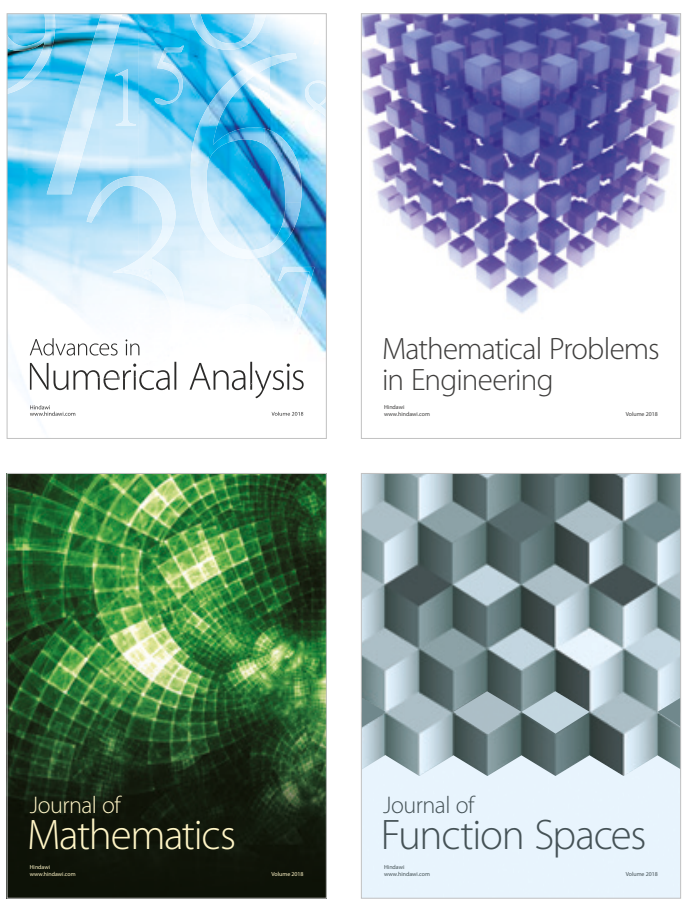

Mathematical Problems in Engineering

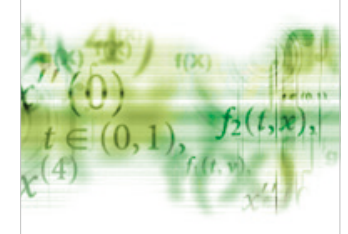

International Journal of

Differential Equations

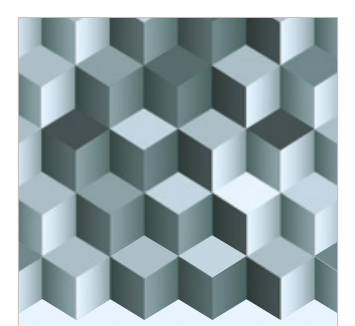

Journal of

Function Spaces

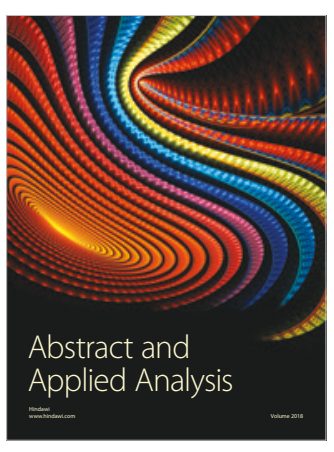

The Scientific

World Journal

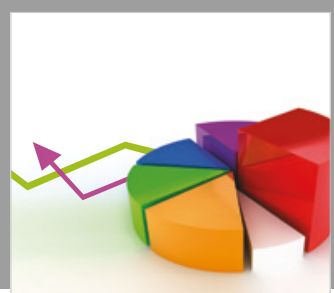

Journal of

Probability and Statistics
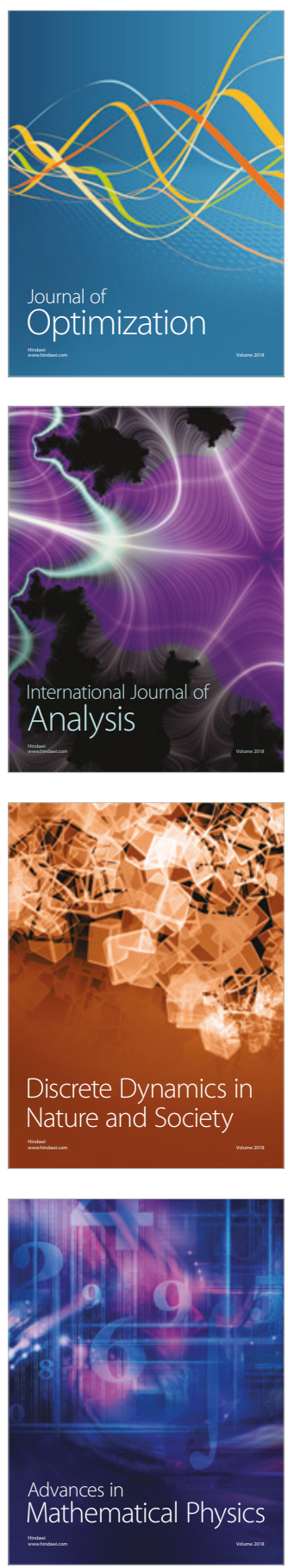